\title{
Surface Plasmon Excitation in Fiber-Optics Sensors: A Novel Theoretical Approach
}

\author{
Ó. Esteban, R. Alonso, M. C. Navarrete, and A. González-Cano
}

\begin{abstract}
A theoretical method for the study of surface plasmon excitation in metallic layers, as used in fiber-optics sensors, is presented. It is based in the calculation of the propagated fields in the waveguide structure and allows us to compute the loss of optical power in the fiber (which is the measured parameter) from energy conservation considerations. The agreement with experimental data obtained with real sensors is good. The method is conceptually simple and can be adapted to different configurations of the sensors.
\end{abstract}

Index Terms-Fiber-optics sensors, surface plasmons.

\section{INTRODUCTION}

I $\mathrm{N}$ recent years, surface plasmon resonance in metallic layers included in waveguide structures has been used as the physical principle for many different fiber-optics devices, including couplers, polarizers, and sensors [1]-[9]. Although varied theoretical methods have been proposed for the study of such phenomena [2], [10]-[15], the results are not always satisfactory, due to different reasons, such as complexity in the calculations, approximations not applicable in every case, and poor agreement with experimental data.

The structure studied here (which is representative of the aforementioned devices) is shown in Fig. 1. A monomode fiber is polished and a metallic layer is deposited onto it. This kind of structure has become common in many sensors because it has been observed that its behavior is strongly dependent on the refractive index of the surrounding medium [2], [8], [11], [16]-[18].

Two main difficulties are present in the theoretical problem of the propagation of light in these structures. First, the cylindrical symmetry of the fiber is not kept in the problem, due to the presence of the deposited plane layer. Practically all of the theoretical models that have been proposed for these structures are based on modal decompositions, which are themselves a major problem because the nonsymmetry of the devices makes these decompositions approximate, even though they are exact for simple structures [2], [10]-[12], [14], [17].

The other main complication is that, due to the presence of a metallic layer, which is absorbent and, therefore, has a complex refractive index, all of the calculations are to be made in the

Manuscript received May 11, 2001; revised November 21, 2001.

Ó. Esteban is with the Departamento de Electrónica, Universidad de Alcalá, Madrid 28040, Spain.

R. Alonso is with the Departamento de Física Aplicada, Facultad de Ciencias, Universidad de Zaragoza, Zaragoza 50009, Spain.

M. C. Navarrete and A. González-Cano are with the Departamento de Óptica, Facultad de C.C. Físicas, Universidad Complutense, Madrid, 28040, Spain (e-mail: agus@ fis.ucm.es).

Publisher Item Identifier S 0733-8724(02)02200-4. complex plane, which is complicated, especially when integral equations are present, which occurs in the most sophisticated models [14].

In this work, we propose a novel theoretical approach, based on the determination of the propagated fields within the structure and in the calculation of the loss of the transmitted optical power, which is the directly measured parameter from the energy balance between the different fields.

The main idea is to begin the calculation with an incident field that corresponds to the evanescent wave propagating in the polished cladding. This field is transferred to the metallic layer, where, eventually, a surface plasmon can be excited for specific values of the refractive index of the outer medium. Whenever a surface plasmon is generated, the transfer of power from the fiber to the metallic layer is critically increased and, therefore, the optical power transmitted by the fiber is significantly decreased. We can determine the loss of optical power by computing the energy associated to the plasmon.

\section{Calculation of the Propagated Fields}

We will take as the incident field the one corresponding to the evanescent wave calculated from the classical treatments of the cylindrical waveguide [19]

$$
\mathbf{E}_{\text {inc }}=\frac{1}{N}\left[\begin{array}{c}
\frac{K_{0}(W R)}{K_{0}(W)} \\
-j \frac{(2 \Delta)^{1 / 2}}{V} W \frac{K_{1}(W R)}{K_{0}(W)}
\end{array}\right] \exp (j \beta z)
$$

where $R$ is the normalized radial coordinate $(r / \rho$, with $\rho$ being the core radius), $n_{\mathrm{cl}}$ and $n_{\mathrm{co}}$ are the refractive indexes of cladding and core, $N$ is a normalization factor corresponding to the total field transmitted in the fiber, $W=\rho \sqrt{\beta^{2}-k_{0}^{2} n_{c l}^{2}}$ is the modal parameter of the cladding, $V=\rho \sqrt{k_{0}^{2} n_{\mathrm{co}}^{2}-k_{0}^{2} n_{\mathrm{cl}}^{2}}$ is the normalized frequency of the fiber $\left(k_{0}\right.$ is the vacuum wavenumber of the incident light and $\beta$ is the propagation constant in the $z$ direction), and $\Delta=1 / 2\left(1-\left(n_{\mathrm{cl}}^{2} / n_{\mathrm{co}}^{2}\right)\right)$ is the step-index factor.

This field corresponds to a fundamental mode polarized along the $x$ axis of the monomode fiber. It is not included in the effect of the perturbation due to the polishing of the cladding or the deposition of the metal layer. In that sense, we must consider (1) as approximate. The intensity distribution corresponding to (1) as a function of the distance of propagation $z$ and the position in the plane of the interface $y$ is shown in Fig. 2. This intensity has been obtained from the values of the polished region of the studied sensors. The dimensions of these regions can be determined directly by measuring the size of the elliptical section of 
Outer medium
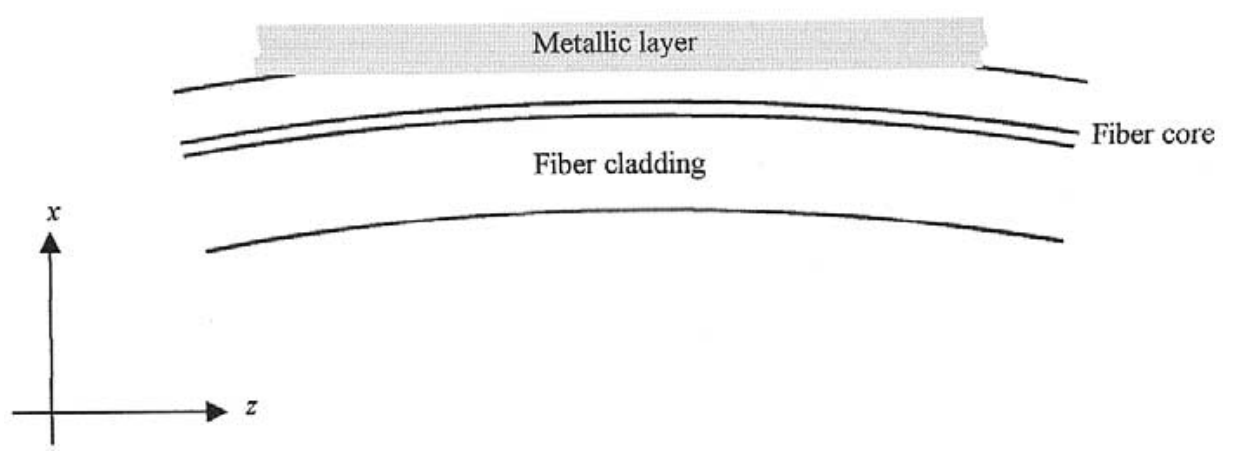

Fig. 1. Scheme of the studied structure. A fiber is polished and a metallic layer is deposited onto it.

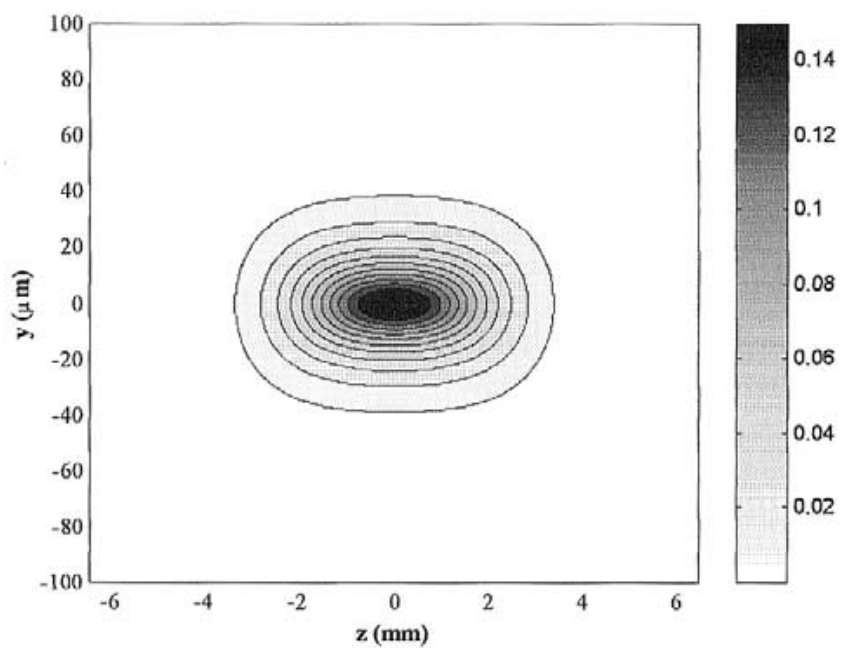

Fig. 2. Intensity distribution of the incident field, corresponding to the evanescent field in the remaining part of the polished cladding, calculated in the interface with the metal. Normalized intensity is encoded in gray levels. Diameter of the fiber core is $9 \mu \mathrm{m}$, curvature radius of the fiber is $192 \mathrm{~mm}$, core refractive index is 1.44868 , and cladding index is 1.44439 . The minimum distance between core and metallic layer (i.e., minimum thickness of the remaining cladding) is $5 \mu \mathrm{m}$.

the polished cladding, where the fields are to be matched [20], [21].

We are interested now in propagating this incident field to the deposited metallic layer. To achieve this, we express the fields in the different regions of the structure as Rayleigh expansions [22]

$$
\begin{aligned}
& \mathbf{E}_{m}(x, y, z)=\frac{1}{4 \pi^{2}} \iint_{-\infty}^{\infty} e^{j(\nu y+\beta z)} \\
& \cdot\left[\mathbf{A}_{m}^{+}(\nu, \beta) e^{j \zeta_{m} x}+\mathbf{A}_{m}^{-}(\nu, \beta) e^{-j \zeta_{m} x}\right] d \nu d \beta .
\end{aligned}
$$

Here, $\mathbf{A}_{m}^{+}$and $\mathbf{A}_{m}^{-}$are the vector Fourier amplitudes of the field in the $m$ th medium ( $m=1,2,3$, corresponding to cladding, metallic layer, and outer medium, respectively), with the signs \pm denoting the fields propagating in the positive and negative $x$ directions and $\nu$ and $\beta$ being the components of the wave vector parallel to the interface, corresponding to the $y$ and $z$ coordinates. The third component of the wave vector (corresponding to the $x$ direction, perpendicular to the interface), which varies in the different layers, can be related to $\nu$ and $\beta$ by

$$
\zeta_{m}=\left[k_{0}^{2} n_{m}^{2}-\nu^{2}-\beta^{2}\right]^{1 / 2}
$$

To calculate the propagated fields (relating the Fourier amplitudes corresponding to the different regions) we must take into account the condition of zero divergence of the fields $\mathbf{E}_{m}$

$$
\begin{aligned}
\zeta\left(\mathbf{A}_{m}^{+}\right)_{x}+\nu\left(\mathbf{A}_{m}^{+}\right)_{y}+\beta\left(\mathbf{A}_{m}^{+}\right)_{z} & =0 \\
-\zeta\left(\mathbf{A}_{m}^{-}\right)_{x}+\nu\left(\mathbf{A}_{m}^{-}\right)_{y}+\beta\left(\mathbf{A}_{m}^{-}\right)_{z} & =0 .
\end{aligned}
$$

and the boundary conditions, which impose the continuity of the tangential component of the fields in the interface. The obtained relationships between the Fourier amplitudes of the field in adjacent regions, expressed as vectors of six components, may be expressed in a matrix form

$$
\left(\begin{array}{l}
\mathbf{A}_{m+1}^{+} \\
\mathbf{A}_{m+1}^{-}
\end{array}\right)=\frac{1}{2 \zeta_{m+1}}\left(\begin{array}{ll}
\mathbf{M}_{m, m+1}^{++} & \mathbf{M}_{m, m+1}^{+-} \\
\mathbf{M}_{m, m+1}^{-+} & \mathbf{M}_{m, m+1}^{++}
\end{array}\right)\left(\begin{array}{l}
\mathbf{A}_{m}^{+} \\
\mathbf{A}_{m}^{-}
\end{array}\right)
$$

for $m=1,2$, where the $3 \times 3$ submatrices $\mathbf{M}_{m, m+1}^{ \pm \pm}$are given by [22] (7)-(10), shown at the bottom of the next page, for $m=$ 1,2 and with $C_{1}=1$ and $C_{2}=\exp \left(j \zeta_{2} d\right)$, where $d$ is the thickness of the layer.

Finally, we impose that the field amplitude corresponding to the field incoming to the layer from the outer medium is zero

$$
\mathbf{A}_{3}^{-}(\nu, \beta)=0
$$

By using these equations, we can determine the propagation of the incident field to the different regions and, in particular, on the upper side of the metallic layer, interface $(m, m+1)=(2,3)$, which is where the long-range surface plasmon may be excited. The short-range plasmon should not, in principle, be excited for the values of the refractive indexes and thickness considered 
in our experiments, because the value of the effective index of the mode guided by the fiber is far from the values of effective indices of short-range plasmons, so we concentrate our study in the long-range one.

The calculation procedure is as follows.

1) We numerically compute, using (1), the values of the normalized incident field for a given set of initial parameters of the structure (refractive indexes and thickness of the core and the cladding that remains after polishing of the fiber and the curvature of the fiber).

2) We Fourier-transform this field by a fast Fourier transform (FFT) algorithm to obtain the Fourier amplitudes of the incident field $\mathbf{A}_{1}^{+}$.

3) We use (6) to propagate the field and to determine the values of the Fourier amplitudes of the field in the different regions, specifically on the metallic layer, $\mathbf{A}_{2}^{+}$and $\mathbf{A}_{2}^{-}$.

4) We then obtain, again with (6), the Fourier amplitude of the field in the outer medium, $\mathbf{A}_{3}^{+}$. Because $\mathbf{A}_{3}^{-}=0$ we impose $\mathbf{M}_{2,3}^{-+}=\mathbf{M}_{2,3}^{--}=\mathbf{0}$.

5) Once we know the Fourier amplitudes, we use an inverse FFT algorithm to determine the field $\mathbf{E}_{3}$ corresponding to the interface $(2,3)$, the upper side of the metallic layer.

6) We calculate the energy corresponding to the field distribution in that surface by averaging the modulus of the field. Then, we subtract this value from the value of the incident intensity. This is the way we can evaluate the attenuation of the incident field, which is the main objective of the calculation.

Fig. 3 shows an intensity distribution corresponding to a surface plasmon generated on the upper side of the metallic layer. A three-dimensional (3-D) view is shown in Fig. 4. This calcu- lation has been performed for a gold layer of 21-nm thickness $(n=0.52+10.7 j)$ deposited onto a monomode optical fiber with a curvature radius of $192 \mathrm{~mm}$ and refractive indexes of core and cladding 1.44868 and 1.44439 , respectively. As can be observed, the field attenuation in the direction of propagation $z$ is smaller than the attenuation in the transverse direction $y$.

\section{EXPERIMENTAL RESULTS}

To check the validity of the presented model, we have compared the obtained results with some experimental measures performed by Alonso et al. [8], [20]. We will also show the performance of this method with respect to other methods of the literature.

Because the behavior of the devices depends on the refractive index of the outer medium, we can use our model introducing different input values for this parameter and computing the energy associated to the field in the metallic layer and, subsequently, the attenuation of the power transmitted by the fiber by following the steps described in Section II.

In Fig. 5, we show the results for a structure with a gold layer of $21 \mathrm{~nm}$ and a thickness of the remaining cladding of $5 \mu \mathrm{m}$. The continuous curve corresponds to the simulation performed by applying our theoretical method and the circles to the experimental results. As can be seen, we can predict the point of maximum energy in the layer, which of course corresponds to the minimum transmission of optical power by the fiber. The ascending part of the curve is also a good adjustment of the experimental values. For the descending part, the adjustment is not as good, which is expected, because the mechanism responsible for the losses of optical power cannot be identified as plasmon excitation, because no plasmon can be excited below a given value

$$
\begin{aligned}
\mathbf{M}_{m, m+1}^{++}= & \left(\begin{array}{ccc}
\frac{\left(n_{m}^{2} \zeta_{m+1}+n_{m+1}^{2} \zeta_{m}\right) C_{m}}{n_{m+1}^{2}} & 0 & 0 \\
\frac{\nu\left(n_{m+1}^{2}-n_{m}^{2}\right) C_{m}}{n_{m+1}^{2}} & \left(\zeta_{m+1}+\zeta_{m}\right) C_{m} & 0 \\
\frac{-\beta\left(n_{m+1}^{2}-n_{m}^{2}\right) C_{m}}{n_{m+1}^{2}} & 0 & \left(\zeta_{m+1}+\zeta_{m}\right) C_{m}
\end{array}\right) \\
\mathbf{M}_{m, m+1}^{+-} & =\left(\begin{array}{ccc}
\frac{\left(n_{m}^{2} \zeta_{m+1}-n_{m+1}^{2} \zeta_{m}\right)}{n_{m+1}^{2} C_{m}} & 0 & 0 \\
\frac{\nu\left(n_{m+1}^{2}-n_{m}^{2}\right)}{n_{m+1}^{2} C_{m}} & \frac{\left(\zeta_{m+1}-\zeta_{m}\right)}{C_{m}} & 0 \\
\frac{-\beta\left(n_{m+1}^{2}-n_{m}^{2}\right)}{n_{m+1}^{2} C_{m}} & 0 & \frac{\left(\zeta_{m+1}-\zeta_{m}\right)}{C_{m}}
\end{array}\right) \\
\mathbf{M}_{m, m+1}^{-+} & =\left(\begin{array}{ccc}
\frac{\left(n_{m}^{2} \zeta_{m+1}-n_{m+1}^{2} \zeta_{m}\right) C_{m}}{n_{m+1}^{2}} & 0 & 0 \\
\frac{-\nu\left(n_{m+1}^{2}-n_{m}^{2}\right) C_{m}}{n_{m+1}^{2}} & \left(\zeta_{m+1}-\zeta_{m}\right) C_{m} & 0 \\
\frac{\beta\left(n_{m+1}^{2}-n_{m}^{2}\right) C_{m}}{n_{m+1}^{2}} & 0 & \left(\zeta_{m+1}-\zeta_{m}\right) C_{m}
\end{array}\right) \\
\mathbf{M}_{m, m+1}^{--} & =\left(\begin{array}{ccc}
\frac{\left(n_{m}^{2} \zeta_{m+1}+n_{m+1}^{2} \zeta_{m}\right)}{n_{m+1}^{2} C_{m}} \\
\frac{-\nu\left(n_{m+1}^{2}-n_{m}^{2}\right)}{n_{m+1}^{2} C_{m}} & \frac{\left(\zeta_{m+1}+\zeta_{m}\right)}{C_{m}} & 0 \\
\frac{\beta\left(n_{m+1}^{2}-n_{m}^{2}\right)}{n_{m+1}^{2} C_{m}} & 0 & \frac{\left(\zeta_{m+1}+\zeta_{m}\right)}{C_{m}}
\end{array}\right)
\end{aligned}
$$




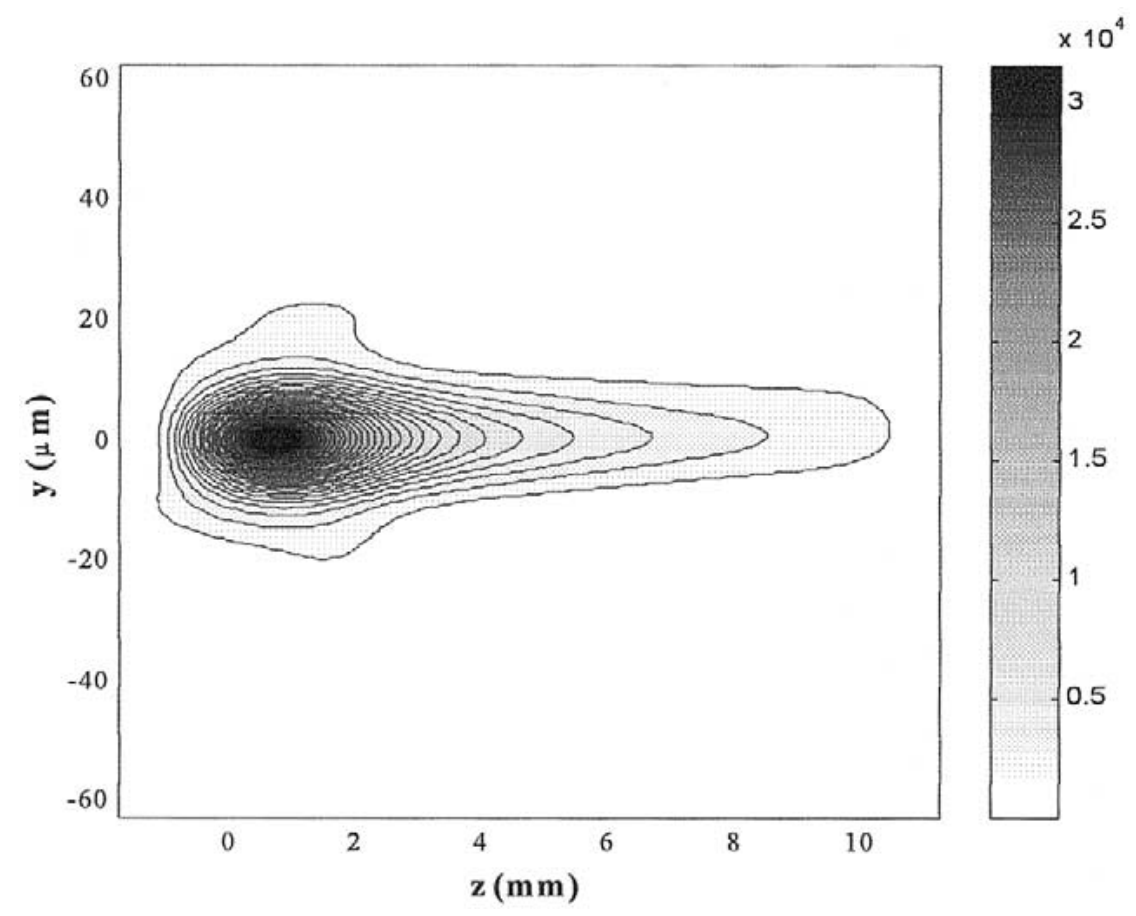

Fig. 3. Intensity distribution (gray-level encoded, arbitrary units) of the field on the upper side of the metallic layer. A plasmon structure is clearly observed. The result is obtained for the data of Fig. 2 and a hold layer 21-nm thick.

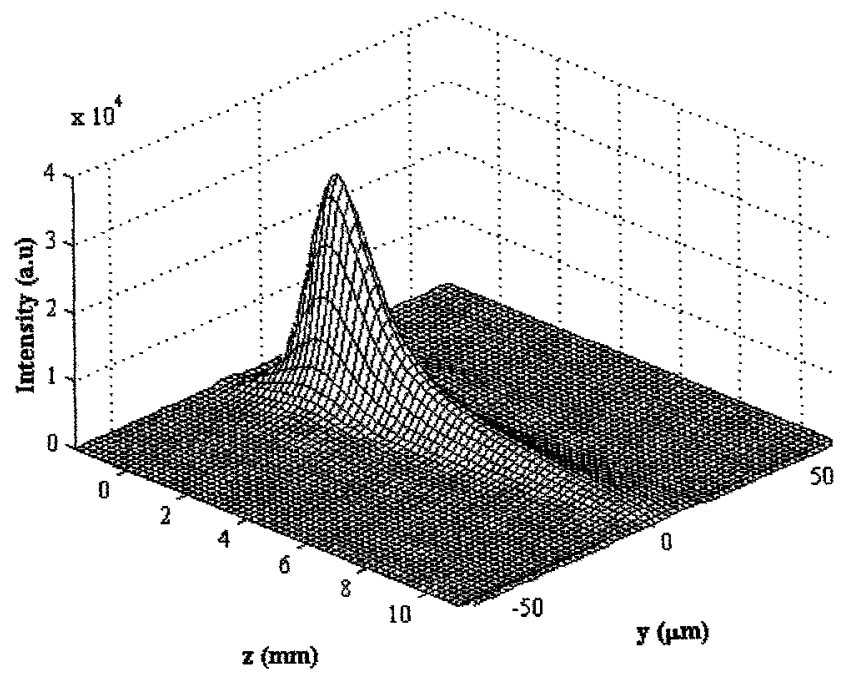

Fig. 4. 3-D view of the intensity distribution of Fig. 3.

of the outer refractive index [1], [8], [20], [23]. However, because the minimum transmission point and the linear increasing region are correctly predicted, our method can be used as a basis for designing devices of this kind that are to be used as sensors, because, in that case, we must adjust the dynamic range of the sensor to the expected values of the outer refractive index.

To compare the performance of our method with other methods, we show the adjustment to the same experimental values of two other theoretical approaches, namely, index matching between modes of the guiding structures [1], [23], [24], (Fig. 6), and perturbative theory [16] (Fig. 7). The index-matching method predicts that the resonance (point of minimum transmittance) appears for the value of the outer refractive index that makes the effective propagation indexes

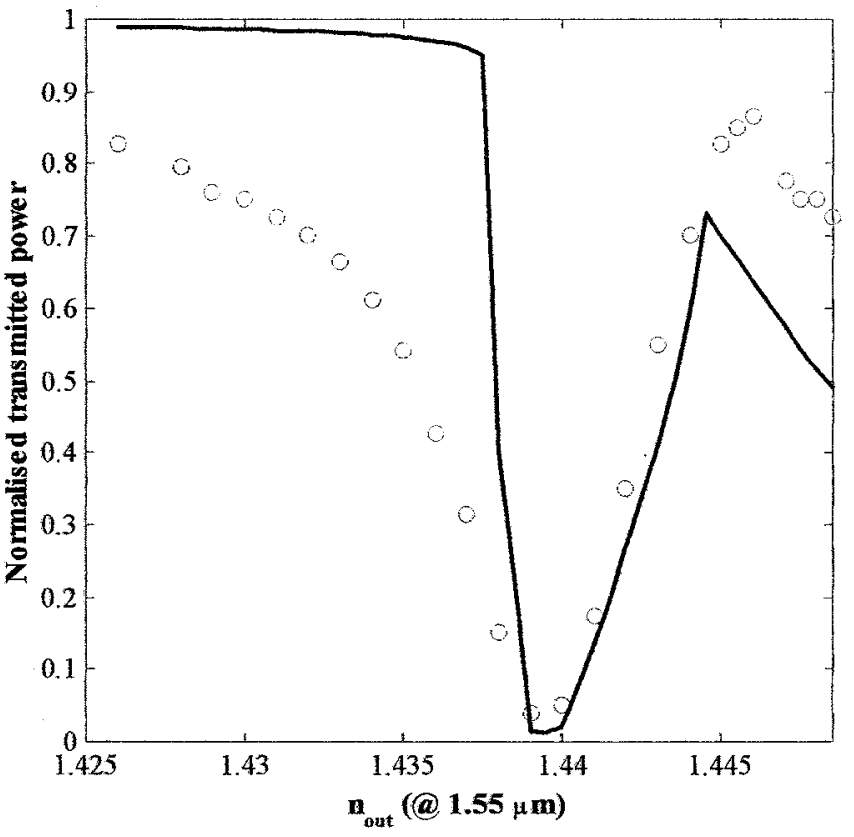

Fig. 5. Comparison between the prediction of the model (solid line) and experimental values (circles) for the dependence of the transmitted power with the refractive index of the outer medium. The data for the structure are those of Figs. 2 and 3.

of the fiber and the mutilayer structures coincident. It can be seen that this predicted value is higher than the one actually occurring in the experiment. Our method permits a better adjustment of the location of the resonance point. On the other hand, the perturbative model, as can be seen in Fig. 7, does not predict accurately the value of the intensity in the resonance, which can be obtained with our technique. In this way, our approach is better suited than the others for the prediction of 


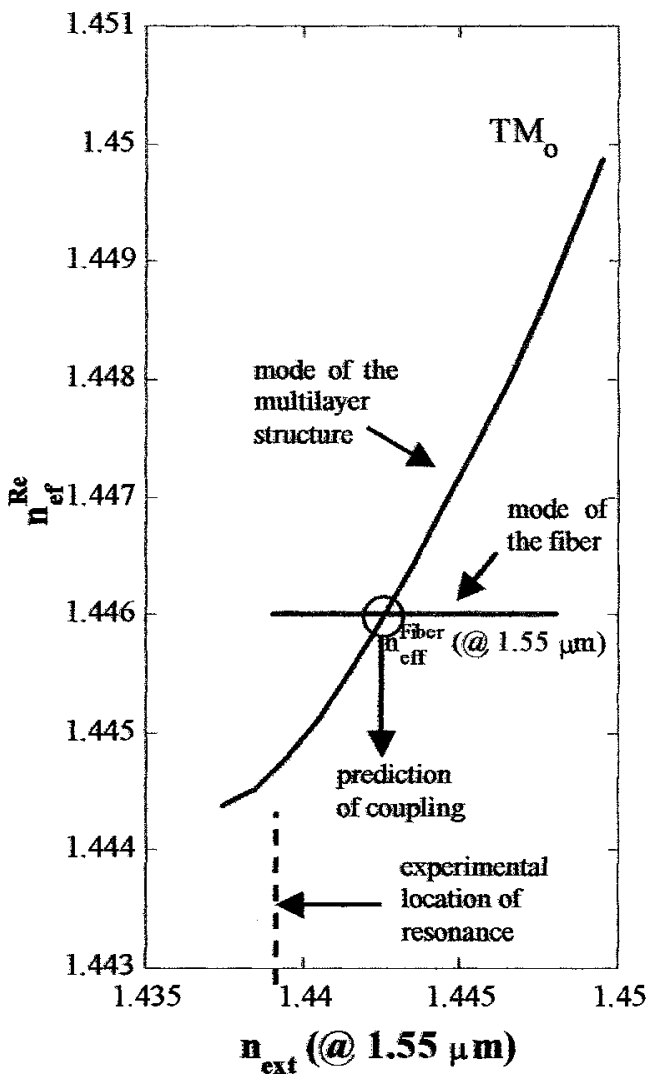

Fig. 6. Prediction of the coupling between modes by the index-matching method. The values of the effective indices for the modes of the structures are represented as a function of the outer refractive index. The experimental resonance point (for the values of Fig. 5) corresponds to a lower value (dashed line) of the outer refractive index than the predicted one.

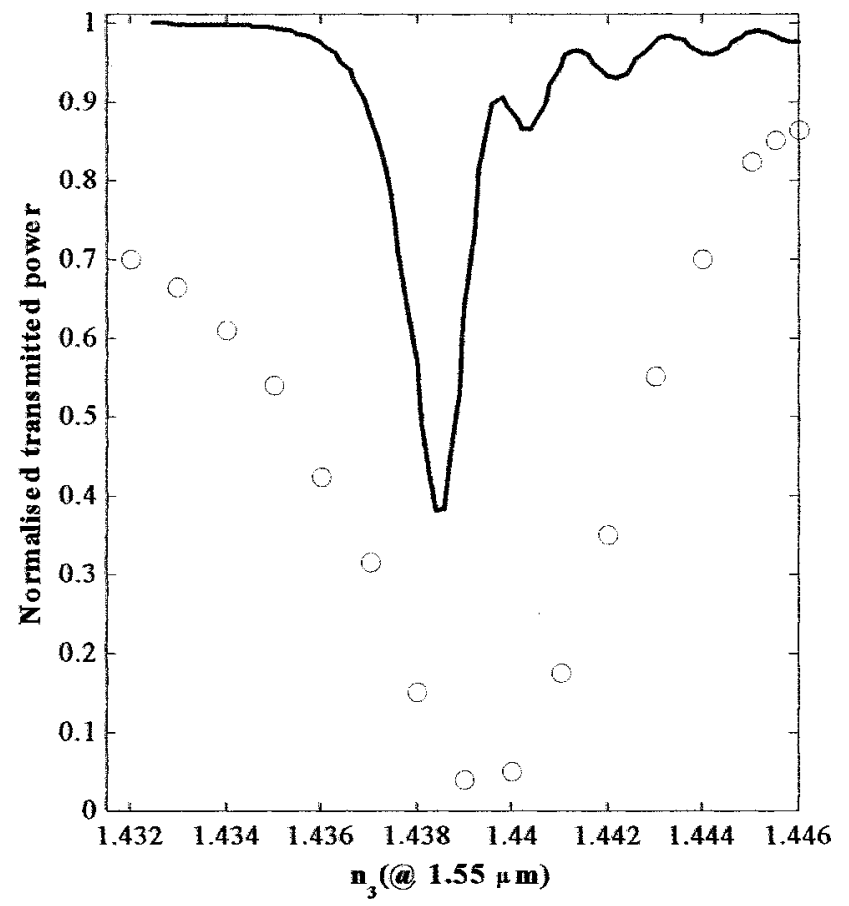

Fig. 7. Comparison between the experimental data (circles) of Fig. 5 and the predictions of the perturbative model.

the behavior of this kind of device because it is needed in the design of the optical fiber sensors based on them.

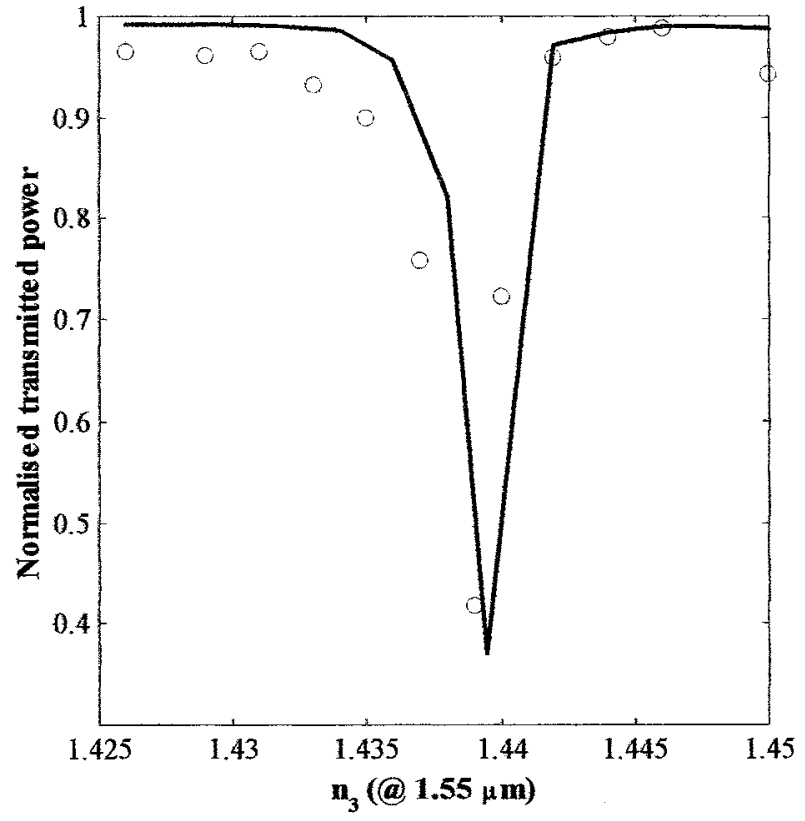

Fig. 8. Comparison between the prediction of the model (solid line) and experimental values (circles) for the dependence of the transmitted power with the refractive index of the outer medium for a thickness for the remaining cladding of $9 \mu \mathrm{m}$.

Finally, we show, in Fig. 8, the results obtained with our method for a different structure, with a gold layer of $21 \mathrm{~nm}$ and a remaining cladding thickness of $9 \mu \mathrm{m}$. As expected, the losses are smaller in the second case because the bigger separation between the core of the fiber and the metallic layer makes the evanescent field reaching the layer weaker. As can be seen, our method permits a good adjustment in this case.

\section{CONCLUSION}

We have presented a novel theoretical approach for the problem of the propagation of fields in waveguide structures consisting on a metallic layer deposited on a polished fiber, showing how surface plasmons can be excited in the layer for given values of the refractive index of the surrounding medium. The algorithms involved are simple and can easily be implemented in any mathematical software package; in our case, we have used MATLAB (MathWorks, Inc., Natick, MA 01760-2098 USA). The procedure can, in principle, be adapted to a wide range of situations, including different indexes and thickness of any number of layers. The agreement with the experimental results is remarkably good in the region of refractive indexes where plasmons are excited and good enough to provide a tool for the design of devices of this kind for different applications.

\section{REFERENCES}

[1] E. Lavretskii, V. Kutsaenko, and W. Johnstone, "Continuous fiber component for optical sensing using multilayer planar overlay with a thin metal film," in Proc. 10th Int. Conf. Optical Fiber Sensors, vol. 2360, 1994 , pp. 557-559.

[2] J. Ctyroký, J. Homola, and M. Skalský, "Modelling of surface plasmon resonance wavguide sensor by couplex mode expansion and propagation method," Opt. Quantum Electron., vol. 29, pp. 301-311, 1997.

[3] R. Slavík, J. Homola, and J. Ctyroký, "Optical fiber surface plasmon resonance sensor for an aqueous environment," in Proc. 12th Int. Conf. Optical Fiber Sensors, vol. 16, Washington, DC, 1997. 
[4] S.-M. Tseng, K.-Y. Hsu, and K.-F. Chen, "Analysis and experiment of thin metal-clad fiber polarizer with index overlay," IEEE Photon. Technol. Lett., vol. 9, pp. 628-630, May 1997.

[5] G. Stewart et al., "Surface plasmon resonances in thin metal films for optical fiber devices," in Proc. Optical Fiber Sensors, Washington, DC, 1988 , pp. $328-331$

[6] M. N. Zervas, "Optical-fiber surface-plasmon-wave polarizers," in Proc. 6th Int.Conf. Optical Fiber Sensors, Berlin, Germany, 1989.

[7] K. Thyagarajan, S. Diggavi, A. K. Ghatak, W. Johnstone, G. Stewart, and B. Culshaw, "Thin metal-clad waveuide polarizers: Analysis and comparison with experiment," Opt. Lett., vol. 15, pp. 1041-1043, 1990.

[8] R. Alonso, F. Villuendas, J. Tornos, and J. Pelayo, "New 'in-line' opticalfiber sensor based on surfaced plasmon excitation," in Sensors Actuators A, 1993, vol. 37-38, pp. 187-192.

[9] K. Nakamura and K. Yoshida, "Special shape fibers and their sensor applications," in Proc. 12th Int.Conf. Optical Fiber Sensors, vol. 16, Washington, DC, 1997.

[10] D. Marcuse, "Investigation of coupling between a fiber and an infinite slab,” J. Lightwave Technol., vol. 7, pp. 122-130, Jan. 1989.

[11] S. Zheng, L.-N. Binh, and G. P. Simon, "Light coupling and propagation in composite optical fiber-slab waveguides," J. Lightwave Technol., vol. 13, pp. 244-251, Feb. 1995

[12] C. Vasallo, "Rigorous theory for modes of optical fibres with cladding limited by a plane," Electron. Lett., vol. EL-22, pp. 944-945, 1986.

[13] A. Sharma, J. Kompella, and P. K. Mishra, "Analysis of fiber directional couplers and coupler half-blocks using a new simple model for single-mode fibers," J. Lightwave Technol., vol. 8, pp. 143-151, Feb. 1990.

[14] M. S. Dinleyci and D. B. Patterson, "Vector modal solution of evanescent coupler,” J. Lightwave Technol., vol. 15, pp. 2316-2324, Dec. 1997.

[15] Ó Esteban, M. C. Navarrete, A. González-Cano, and E. Bernabeu, "Analysis of the behavior of compound waveguide structures used as fiber-optic sensors," Opt. Lasers Eng., vol. 33, pp. 219-233, 2000.

[16] R. Alonso, J. Subias, J. Pelayo, F. Villuendas, and F. Tornos, "Single-mode optical-fiber sensors and tunable filters based on the resonant excitation of metal-clad modes," Appl. Opt., vol. 33, pp. 5197-5201, 1994

[17] A. T. Andreev and K. P. Panajotov, "Distributed single-mode fiber to single-mode planar waveguide coupler," J. Lightwave Technol., vol. 11, pp. 1985-1989, Dec. 1993
[18] Ó Esteban, M. C. Navarrete, A. González-Cano, and E. Bernabeu, "Measurement of the degree of salinity of water with a fiber-optic sensor,' Appl. Opt., vol. 38, pp. 5267-5271, 1999

[19] A. W. Snyder and J. D. Love, Optical Waveguide Theory. New York: Chapman \& Hall, 1983

[20] R. Alonso, "Estudio teórico y experimental de dispositivos Ópticos basados en el acoplamiento entre el modo guiado por una fibra Óptica y estructuras multicapa incluyendo medios metálicos," Ph.D. dissertation, Universidad de Zaragoza, Zaragoza, Spain, 1995.

[21] O. G. Leminger and R. Zengerle, "Determination of single-mode fiber coupler design parameters from loss measurements," J. Lightwave Technol., vol. LT-3, pp. 746-754, 1985.

[22] F. I. Baida, D. Van Labeke, and J. M. Vigoureux, "Theoretical study of near-field surface plasmon excitation, propagation and diffraction," Opt. Commun., vol. 171, pp. 317-331, 1999.

[23] B. Culshaw and J. Dakin, Eds., Optical Fiber Sensors: Systems and Applications. Boston: Artech, 1989, vol. II, pp. 625-634.

[24] F. Villuendas, "Excitación y detección en infrarrojo próximo de plasmones superficiales en una intercara metal-dieléctrico. Aplicación en un sensor de fibra óptica," Ph.D. dissertation, Universidad de Zaragoza, Zaragoza, Spain.

Ó. Esteban, photograph and biography not available at the time of publication.

R. Alonso, photograph and biography not available at the time of publication.

M. C. Navarrete, photograph and biography not available at the time of publication.

A. González-Cano, photograph and biography not available at the time of publication. 